\title{
Force Prediction in Robotized Ultrasonic Sheet Metal Forming Based on Finite Element Analysis and Artificial Neural Networks
}

Vytautas Ostasevicius ( $\nabla$ vytautas.ostasevicius@ktu.lt )

Kaunas University of Technology

Agne Paulauskaite-Taraseviciene

Kaunas University of Technology

leva Paleviciute

Kaunas University of Technology

Vytautas Jurenas

Kaunas University of Technology

Paulius Griskevicius

Kaunas University of Technology

Darius Eidukynas

Kaunas University of Technology

\section{Laura Kizauskiene}

Kaunas University of Technology

\section{Research Article}

Keywords: incremental sheet forming, robotised manufacturing, force prediction, artificial neural networks, friction force reduction

Posted Date: September 22nd, 2021

DOI: https://doi.org/10.21203/rs.3.rs-903962/v1

License: (c) (1) This work is licensed under a Creative Commons Attribution 4.0 International License. Read Full License 


\title{
Force prediction in robotized ultrasonic sheet metal forming based on finite element analysis and artificial neural networks
}

\author{
Vytautas Ostasevicius $^{1 * *}$, Agne Paulauskaite-Taraseviciene ${ }^{2}$, Ieva Paleviciute ${ }^{3}$, Vytautas Jurenas $^{4}$, Paulius \\ Griskevicius $^{5}$, Darius Eidukynas ${ }^{6}$, Laura Kizauskiene ${ }^{7}$
}

\author{
Institute of Mechatronics, Kaunas University of Technology, Studentu 56-122, Kaunas LT-51424, Lithuania; vytautas.ostasevicius@ktu.1t \\ Department of Applied Informatics, Kaunas University of Technology, Studentu 50-214, Kaunas LT-51368, Lithuania; E-mail: \\ agne.paulauskaite-taraseviciene@ktu.lt \\ Institute of Mechatronics, Kaunas University of Technology, Studentu 56-105, Kaunas LT-51424, Lithuania; ieva.paleviciute@ktu.lt \\ Institute of Mechatronics, Kaunas University of Technology, Studentu 56-107, Kaunas LT-51368, Lithuania; vytautas.jurenas@ktu.1tu \\ Department of Mechanical Engineering, Kaunas University of Technology, Studentu 56-301, Kaunas LT-51424, Lithuania; \\ paulius.griskevicius@ktu.lt \\ Institute of Mechatronics, Kaunas University of Technology, Studentu 56-125, Kaunas LT-51424, Lithuania; darius.eidukynas@ktu.1t \\ Centre of Real Time Computer Systems, Kaunas University of Technology, Barsausko 59-51423, Kaunas LT-51368, Lithuania; E-mail: \\ laura.kizauskiene@ktu.lt \\ * Correspondence: vytautas.ostasevicius@ktu.lt Tel.: +370 37300909
}

\begin{abstract}
The forces acting in the process of single point incremental forming (SPIF) change the geometry of the sheet metal. The tool-workpiece interaction process is non-linear due to the large deformations of the sheet metal, which determine the plastic behaviour, as well as the evolutionary boundary conditions resulting from the contact between the tool and the sheet. Instead of lubricating the contact surface of the forming tool and the sheet metal, an innovative environmentally friendly method to reduce the coefficient of friction by vibrating the sheet has been proposed. The finite element method (FEM) allowed a virtual evaluation of the deformation parameters of the SPIF process in order to determine the destructive loads. The FEM was chosen as a deterministic numerical tool to evaluate the set of defect parameters induced by forming forces. The paper also proposes a method for predicting the formation force using an artificial neural network (ANN), assuming that such a model is generalized to implicit data. In this context, an empirical analysis of the implementation of the ANN technique is performed.
\end{abstract}

Keywords: incremental sheet forming, robotised manufacturing, force prediction, artificial neural networks, friction force reduction

\section{Introduction}

Robotic SPIF, where a small-sized tool deforms a sheet of metal, is ideal for replacing expensive stamping processes, assuring flexibility, cost-effectiveness as well as increasing the competitiveness of companies. This technology opens up new possibilities compared to traditional small series production methods, where high added value is determined by skills and qualification. However, today's robotic designs are not rigid enough, so the power-enhancing capabilities are associated with additional vibrational excitation. Various research methods have been used to evaluate the mechanical features of the robotized SPIF process [1-3]. The common objective of the experiments is to analyse the results of using an industrial robot with respect to the potential increase in technological capabilities (5-axis production) and process versatility (possibility to perform additional flanges or complete work) compared to traditional 3-axis machining. The articles $[2,3]$ present an approach to link FEM analysis with the modelling of the elastic robot structure to improve the geometric accuracy of the formed metal sheet. During the forming process, the forces at the tool-sheet interface are evaluated and used as input to the elastic robot model [2]. The importance of three numerical and material parameters on the predicted forces is investigated in order to select the most effective tool path correction. The behaviour of the ultrasonically excited forming tool in the SPIF process has been investigated in $[4,5]$. The FEM model was employed to evaluate the influence of the ultrasonic vibration frequencies and amplitudes on the accuracy of the technological process. The results showed that the effect of vibrations on the metal stress, deformation and thickness is minimal at a frequency of $15 \mathrm{kHz}$ and that the vibrations can prevent sheet metal breakage and improve the surface quality of the material. However, increasing the excitation frequency above $40 \mathrm{kHz}$ resulted in a significant increase in axial force.

In order to reduce the computational time in SPIF simulations, the FEM mesh is composed of several non-overlapping parts subjected to plastic and elastic deformations [6]. The plastic deformations are localized, including two substructures: (1) plastic - non-linear and (2) the elastic - pseudo-linear. The group of plastic structures creates a finite element mesh that is in contact with the tool used to form the material. They are gradually updated to take into account any nonlinearities. The remaining finite elastic deformations of the mesh are modelled by elastic base structures, considering that the behaviour is linear. The process is defined by stiffness matrices and internal force vectors, which are measured at the beginning of each iteration and then statically condensed to remove internal degrees of freedom. The deformation limits that can be achieved for the SPIF features are much higher compared to conventional features. Studies based on Marciniak-Kuczynski (MK) theory have shown that the MK damage criterion can be used to predict the SPIF limits for arbitrary loading paths. An analytical process is required to investigate the extent to which such monoform strain paths 
can be induced by highly non-monotonic deformation pathways [7]. The MK model can be used to predict the onset of necking when strain paths have been obtained by FEM modelling. This discrepancy is allocated to the deformation gradient above the thickness of the metal sheet, which is not included in the mathematical model. The stress rate of plastic output and the resistance to deformation that many metallic materials exhibit affect the forces, energies and forming limits associated with rapid forming processes. The effect of stress rate on the forming parameters of different types of steels is examined in [8]. The impact of stress rate on the stress-strain curves of the materials has been experimentally evaluated, and the results obtained, together with the results of the static tensile experiment, have been used to model the constitutional behaviour of the investigated steels by means of the exceptional Johnson-Cook and Voce models, which enable the dynamic modelling of the forming processes. The results revealed that the impact of the stress rate on the forces and energies used in the forming process and their limits are insignificant and highly material dependent.

Materials such as aluminium alloys are widely used for manufacturing automotive, aerospace and other components due to its good strength, low weight and formability. Therefore, the peculiarities of SPIF processes of these alloys have been extensively studied by researchers [9-12]. The SPIF process, as an easy modifiable manufacturing technology, that does not require dies, clearly enhances the formability of the sheet metal. Based on the simplified proportional load assumption, the fracture boundary model, in contrast to the conventional neck, can represent the failure mode according to the AA1050-H111 failure mechanism [9]. The SPIF process uses elastic fracture criteria applied to the generalized tensile stress state based on the degree of triaxiality of the stresses. By combining the appropriate plastic forming principle with the stress elements expressed as process parameters, the proposed modified forming limit model reveals the influence of the process variables, in good agreement with the experimental results. The formation height is also considered as a SPIF parameter. The modified fracture formation boundary model has been shown to be suitable for practical application in damage assessment. SPIF outperforms conventional forming process methods as has advantages such as higher formability achieved by the formed material. Various studies have been carried out and hypotheses have been put forward to interpret this phenomenon, but there is still a lack of studies to substantiate the relationship between the specific stress and tension caused by SPIF and the degradation of the material leading to localization and damage. The research presented in [10] explains the systematic progress of SPIF formation and damage and the extended Gurson Tvergaard Needleman (GTN) model is used to predict surface damage. In order to predict failures, several SPIF cone simulations different wall angles have been performed, and the results showed that the GTN model underestimates the SPIF failure angle due to incorrect fusion modelling. A physically based Thomason merger criterion can be used to improve the prediction results by postponing the start of the merger. The AlMgSc alloy, well known in the field of aeronautics, has been investigated in [11].

A multiple experiment was carried out, where material laws and corresponding material variables were selected and adjusted to correctly define the behaviour of the material. Conical structures were formed in order to determine the maximum forming angle. A mathematical model was developed to confirm that the evolution of the force during the process and the resulting geometrical structure can be predicted. In order to define the deformation behaviour of sheet metal in real production, it is essential to accurately determine the ultimate deformations of the SPIF compared to the traditional material formation limits and the effect of the process parameters on these deformations [12]. In addition, the force of the SPIF is very important, especially when designing special equipment or ensuring that all safety regulations are observed when using adapted machines.

Although SPIF process methods have been extensively researched, significant efforts are needed to increase their applicability to industrial processes. Due to the non-linearity, complexity of the relationship and time-consuming process, the application of statistical methods and AI approaches is beneficial [13-17]. In particular, it is necessary to define the possibility to avoid failures in the design of complex shaped products [13] and to identify factors affecting process efficiency when a cutting section is required. The ANN algorithm can be used to predict failures of complex shaped products in the SPIF process. The SPIF process is limited by the maximum wall angle and the reduced surface quality of the formed material parts [14]. In order to ensure the reduction of surface stiffness and to optimize the forming wall angle of the Al5052 alloy sheet, common material formation variables such as vertical step size, lubrication, tool diameter, and feed rate were determined using genetic algorithms. The study presented in [15], explores capabilities of ANN to predict the minimum force required to perform SPIF on thin sheets made from aluminium AA3003-O and calamine brass Cu67Zn33 alloys. Accordingly, machining variables such as step depth, tool feed rate, tool rotation speed, wall angle, sheet metal thickness, and material type were selected as input parameters to predict the output - minimum vertical force component. The study [16] takes into account certain steps of the sheet metal SPIF process that allow the production of individual components. However, the manual forming process was automated by an advanced implementation. ANN based prediction can be used to estimate the average surface roughness and the maximum forming angle when forming AA5052-H32 material [17]. It has been found that the successful implementation of the ANN model in the shaping process can reduce engineering efforts.

SPIF is a very important task for engineers to anticipate the process in which many independent parameters operate simultaneously. A review of the research results suggests that alternative methods to reduce the coefficient of friction between the tool and the metal sheet contact surfaces have not yet been proposed, the dynamics of the process has not been sufficiently investigated, and there is a lack of physical evidence that virtual models are appropriate. 


\section{Evaluation methodology of forming process}

Metallic materials used in the deforming process, such as single point incremental forming (SPIF), visibly deform on contact with the tool area. The key challenge of the SPIF process is to effectively assess the shaping forces. The forces generated during SPIF may be controlled by varying the coefficient of friction between the tool and the workpiece. Lubrication is an important factor in the SPIF process, reducing friction in the tool-workpiece contact area, but the use of grease is associated with environmental problems. Therefore, it is necessary to find other ways to reduce the shaping forces associated with the process dynamics. This requires, in particular, the development of a physically adequate mathematical model of the process, whose mechanical parameters can be adjusted experimentally using the 3D scanning device shown in Fig. 1.

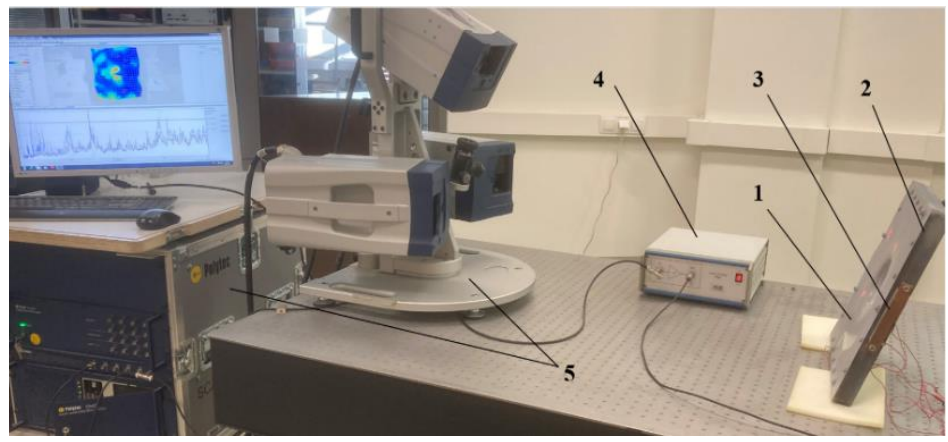

Fig. 1 3D scanning set-up: 1 - an experimental body - aluminum alloy sheet; 2 - steel base frame; 3 - piezoelectric actuator; 4 - liner amplifier P200 (FLC Electronics AB, Sweden), 5 - 3D scanning vibrometer PSV-500-3D-HV (Polytec GmbH, Germany)

A 300x300x0.5 aluminium alloy AW1050 sheet embedded in a 30x30 mm welded steel frame was formed. The frequencies of the resonant modes were determined using the Comsol Multiphysics finite element method. In an attempt to change the lubrication of the contact surface between the forming tool and the sheet metal, an attempt was made to excite the sheet metal by vibrations. For this purpose, equipment, which scheme is presented in Fig. 2, was developed to excite 3D ultrasonic frequency vibrations in SPIF sheet metal.
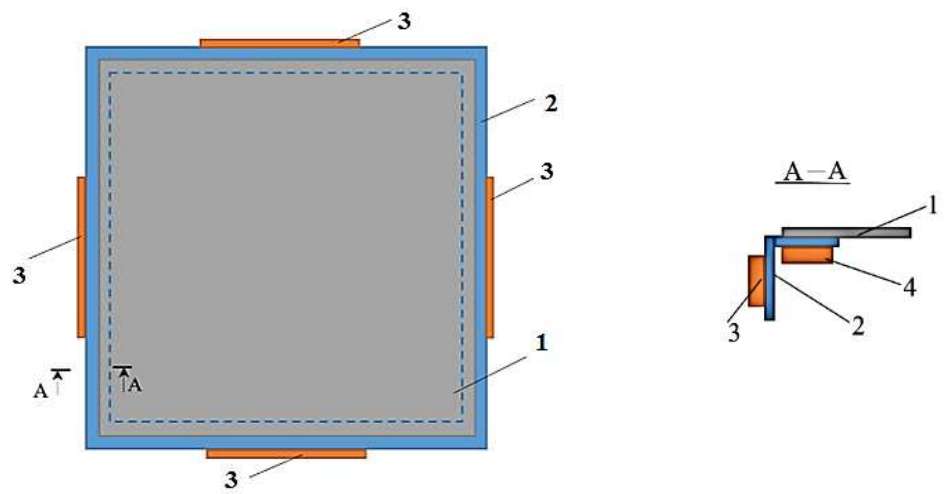

Fig. 2. Scheme of excitation of ultrasonic 3D vibrations in the sheet: 1 - metal sheet; 2 - frame; 3 and 4 - bimorph type piezoelectric actuators

This equipment consists of an angled profile metal frame 2, to which piezo ceramic elements 3 and 4 are glued in different planes, to excite bending oscillations of the higher harmonics in the frame. Since the sheet metal is rigidly attached to the frame by its outer contour, the vibrations of the frame also excite the sheet metal. This principle of ultrasonic 3D vibrations excitation in the sheet metal makes it possible to reduce the friction between the sheet metal and the tool during SPIF, to improve the lubrication conditions at the tool-workpiece contact pair, and in some cases, to completely eliminate the lubrication. From 2 to 8 piezo ceramic elements in the rectangular can be used to excite the 3D vibrations in the sheet metal according to the given scheme (Fig. 2) by alimenting them with harmonic ultrasonic frequency electrical signals. Experimental studies on a $0.5 \mathrm{~mm}$ thick aluminium plate show that the maximum reduction in friction forces at the tool-workpiece contact pair was observed when the piezoelectric actuators were excited in the 25$35 \mathrm{kHz}$ frequency range. The sheet metal vibrational analysis with the Polytec scanning vibrometer PSV-500-3D-HV, presented in Fig. 1, shows that in this frequency band the workpiece is dominated by planar (XY) higher harmonic vibrations, which are significantly less suppressed in the tool-sheet metal pair friction contact than in the perpendicular to the metal sheet direction $\mathrm{Z}$ (Fig. 3). These results show that when two piezoelectric actuators in different planes are excited, the in-plane (XY) oscillations in the $30-33 \mathrm{kHz}$ band are greatly enhanced. 


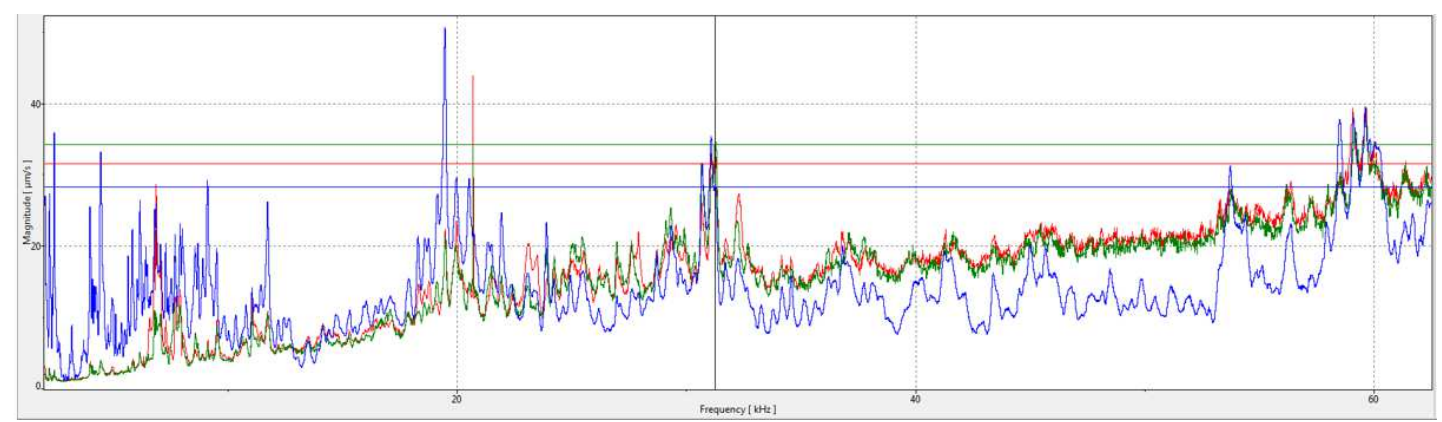

(a)

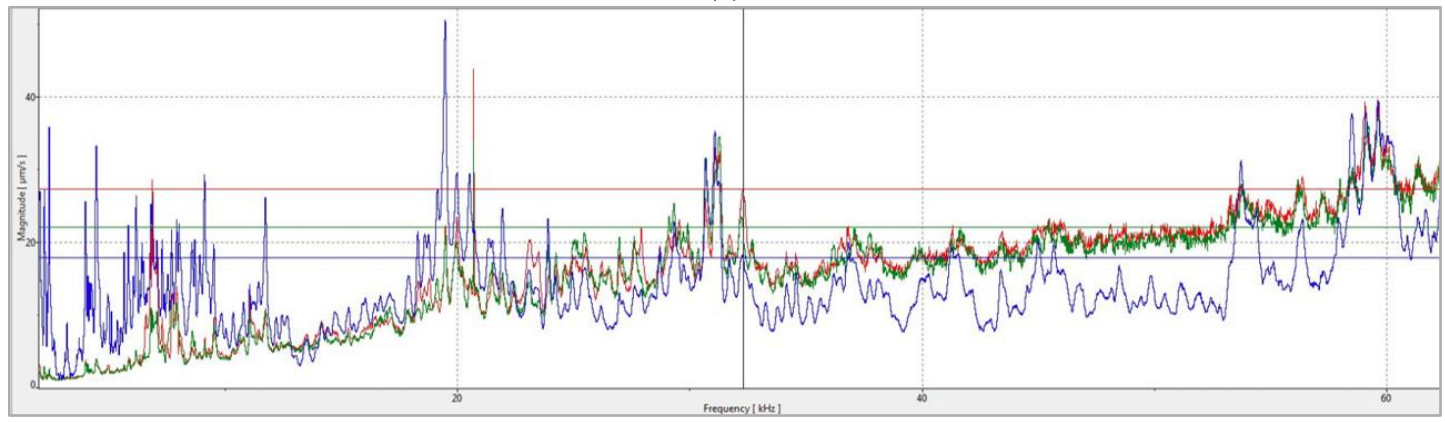

(b)

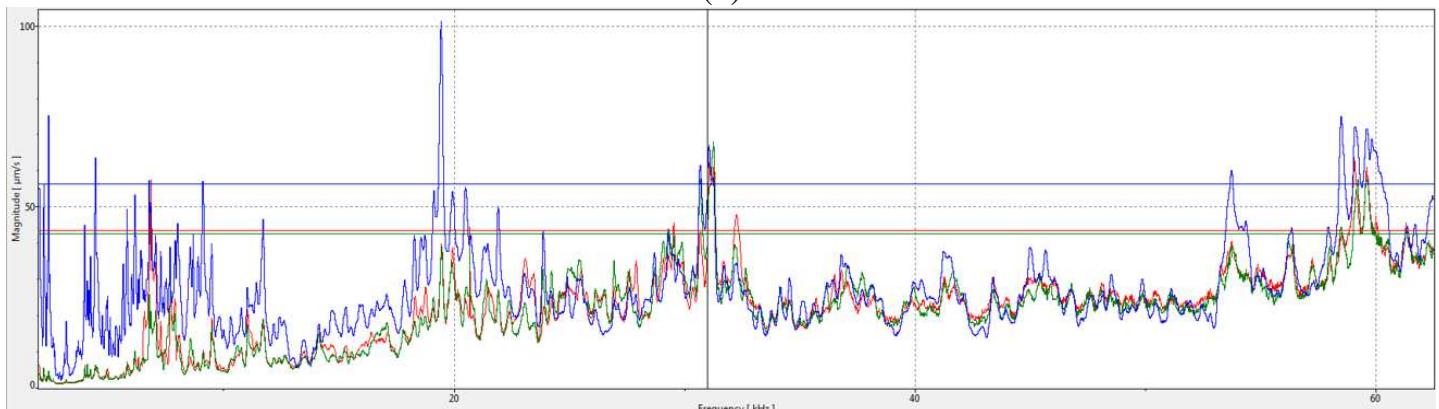

(c)

Fig. 3 The amplitude-frequency characteristics of the sheet metal measured with a Polytec 3D scanning vibrometer in the frequency range from $2 \mathrm{kHz}$ to $60 \mathrm{kHz}$ after excitation of two differently arranged piezoelectric actuators (according to Fig. 2): a actuator 4 is excited, $\mathbf{b}$ actuator 3 is excited, $\mathbf{c}$ both actuators are excited, where $X$ (red) and $Y$ (green) are lateral vibrations, $Z$ (blue) are vibrations in normal direction to a sheet respectively

In order to assess the effect of vibrations on the SPIF of an aluminium alloy sheet, measurements of the surface roughness of the sheet were made with and without ultrasonic vibrations. An advanced surface roughness tester (TR200 Time group) was used to measure the roughness. The measured surface roughness of the sheet formed without ultrasonic vibrations warried in range $R_{a}=0.30-0.33 \mu \mathrm{m}$ and with the ultrasonic vibrations in range $R_{a}=0.18-0.25 \mu \mathrm{m}$, respectively.

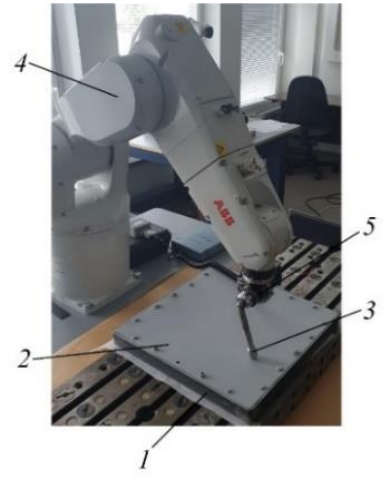

(a)

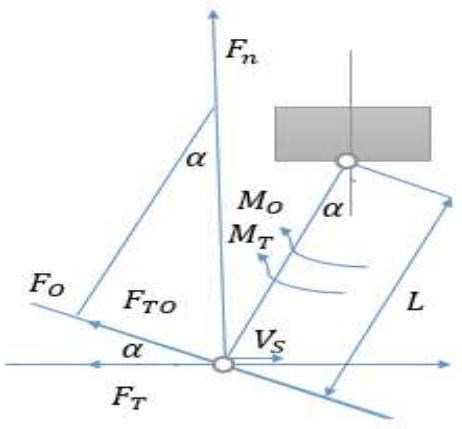

(b)

Fig. 4 Determination of friction force: (a) interaction and (b) calculation scheme of single point tool and aluminium alloy sheet: 1 - frame for sheet, 2 - aluminium sheet, 3 - forming tool, 4 - Robot ABB IRB1200, 5 - mechanical (friction) torque meter STJ100 
In order to determine the frictional force between the tool and the aluminium alloy sheet (Fig. 4, a), calculations were carried out in accordance with the diagram in Fig. 4, b.

Since the roughness momentum gauge was placed with an angle of $30^{\circ}$ degrees, both pressure and friction torque could be obtained. The friction force can be calculated as follows:

$$
\begin{gathered}
F_{T}=k_{T} F_{N} . \\
F_{T O}=F_{T} \sin \alpha . \\
M_{O}=F_{O} L . \\
F_{N}=\frac{F_{O}}{\sin \alpha} . \\
M_{O}+M_{T}=\left(F_{O}+F_{T O}\right) \cdot L .
\end{gathered}
$$

where $\mathrm{L}$ - the length of the tool link connecting sphere and moment gauge $(150 \mathrm{~mm}) ; \alpha$ - an angle between the sample sheet surphase and the tool link $\left(30^{\circ}\right) ; M_{O}, M_{T}$ - tool pressing force and tool friction force generated angular momentums; $F_{O}, F_{T O}$ - tool priessure force and the friction force projection to tools link; $F_{N}, F_{T}$ - tool's sphere pressure and friction force; $k_{T}$ - friction force cofficient between the sample sheet and the steel tool sphere; $v_{s}-$ steel tool's sphere speed on the surface of the sample sheet $(\mathrm{mm} / \mathrm{s})$.

The mechanical (friction) torque meter STJ100 (BGI series digital force gauge with torque sensor STJ100, Mark-10 Corp., USA) measured that the sensitivity factor was $6 \mathrm{Nm} / \mathrm{V}$ during the experiment. The results of the tool friction coefficient and friction force measurements on dry, lubricated and ultrasonically excited surfaces in the $30-33 \mathrm{kHz}$ frequency range, which reduces the friction force at the tool-sheet metal contact pair, are shown in Table 1.

Table 1 Friction coefficient and friction force measurement results

\begin{tabular}{lcc}
\hline \multicolumn{1}{c}{ Method } & Friction Force & Friction coefficients \\
\hline Without lubrication and vibration & $3,2 \mathrm{~N}$ & 0,5 \\
With lubrication & $1,6 \mathrm{~N}$ & 0,1 \\
With vibration & $1,9 \mathrm{~N}$ & 0,12 \\
\hline
\end{tabular}

The Table 1 shows that the coefficient of friction between the steel tool and the aluminium alloy sheet is close to that of the lubricated surfaces when subjected to ultrasonic vibrations. This makes it possible to solve environmental problems, and the surface of the manufactured part does not need to be cleaned, while at the same time its roughness is reduced by half. This revealed phenomenon has been patented by the authors [18].

\section{Mechanical testing of aluminium alloy sheet}

The complexity of SPIF mechanics and the special conditions, such as bending under stress, cyclic bending and unbending, as well as the shear deformation process, which contribute to the improvement of the overall formability, require research efforts and techniques. The SPIF has much higher forming limits than those achievable by conventional sheet forming processes such as deep drawing. Major parameters affecting the formability are the tool size, the step down (drawing angle), the thickness and the material properties of the sheet metal. The formability increases with decreasing tool size, step down per revolution and thickness of the sheet, while the feed rate do not have a significant effect [19]. The strain-based forming limit curve criterion is widely used in the sheet metal forming industry to predict fracture. This criterion is only valid when the strain path is linear [20]. In contrast, during the SPIF process the strain path is rather nonlinear and the practice of using a strain-based forming limit criterion often leads to an erroneous assessment of formability and failure prediction. Stress-based forming limits are not sensitive to any strain path changes and are used to model the necking limit, combined with the fracture limit based on the criterion of maximum shear stress [21]. The strain path, the stress state and the prediction of the formability of the SPIF were evaluated by FE analysis. In order to develop a validated numerical model of the SPIF process, several sets of experimental tests were performed. Firstly, the material model was calibrated using experimental results of the cupping test. Subsequently, the same material model was used for the SPIF simulation. In order to characterize the plastic behaviour and ductile fracture of the aluminium alloy sheet, the Erichsen cupping tests on square aluminium plates were performed in accordance with ISO 20482: 2013 (Metallic materials - Sheets and strips - Erichsen tensile test). The Erichsen standard provides information on fracture under the equi-biaxial state of stress. The most highly stressed point is located at the apex of the dome where the components of radial and circumferential stress and strain are equal to each other. The main mechanical characteristic obtained from the cupping test is the Erichsen Index (IE), which refers to the depth of the sphere at the maximum force, and is of relatively minor importance in assessing the plasticity of the material. The results are influenced by the sheet thickness and the friction between the sheet and the tool surface. For the Erichsen compression test, the specimen was attached to a $55 \mathrm{~mm}$ 
die with a heavy flange and bolts tightened to achieve a force of approximately $10 \mathrm{kN}$ on the workpiece holder (Fig. 5). The radius of the hemi-spherical punch simulating the forming tool was $10 \mathrm{~mm}$ and the test speed was $5 \mathrm{~mm} / \mathrm{min}$.

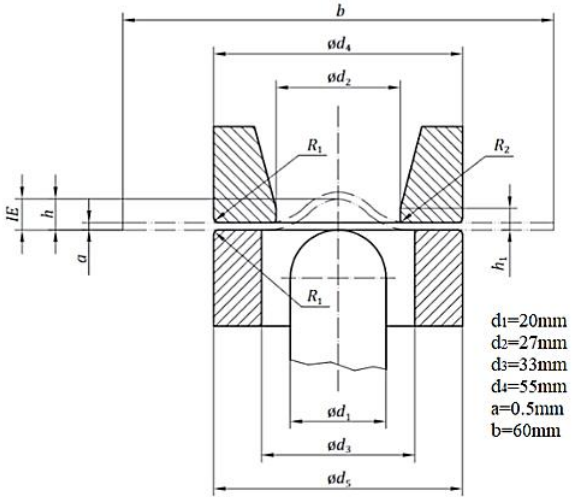

(a)

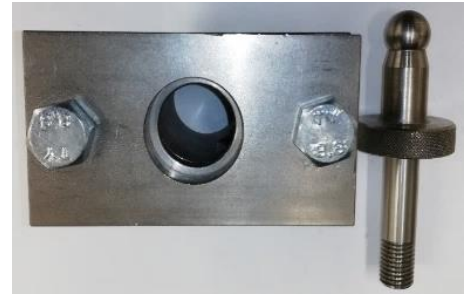

(b)

Fig. 5. Erichsen cupping test: (a) scheme, (b) test die.

The force displacement response was obtained by plotting the reaction force on the punch vs the displacement of the punch (Fig. 6).

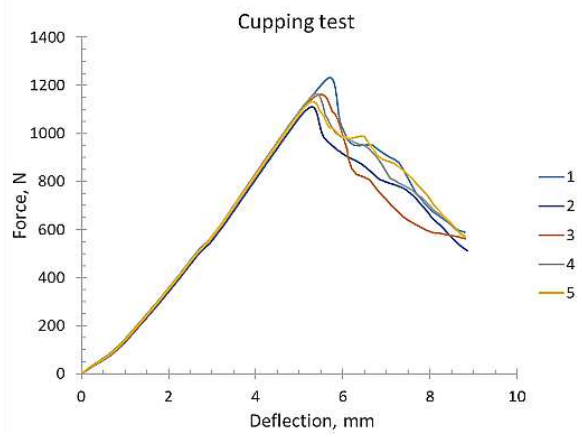

(a)

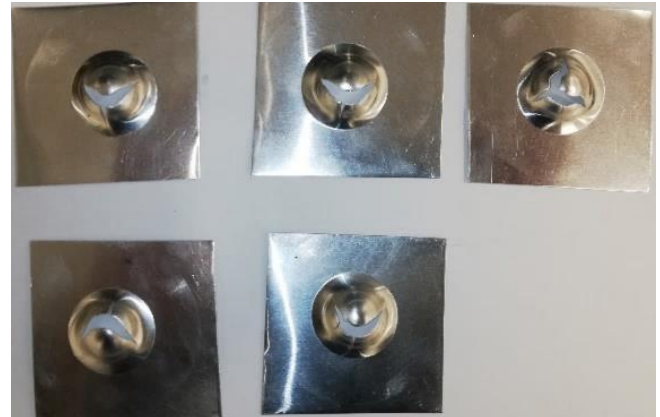

(b)

Fig. 6. Erichsen cupping test results: (a) cupping curves for specimens 1-5 and (b) nature of failure of the specimens 1-5 after the cupping test

The experiment was carried out by applying a hemispherical punch to a sheet of metal until a crack appeared, while simultaneously measuring the depth of the cup as indicated by a circular scale. A harder metal with a fine grain structure will have a shallower depth and a fine shape of a cup. On the other hand, a ductile metal, with a coarse grain size, will produce a deeper indent with a rough cup shape. The maximum load for all 5 cupping tests was $F_{\max }=1160 \mathrm{~N}$, Erichsen index $I E=5.36 \mathrm{~mm}$. The force vs displacement curve was chosen as the validation criterion for the numerical model. A $0,5 \mathrm{~mm}$ thick sheet of aluminium alloy EN AW1050A H24 was used for the analysis. The properties of the material are presented in Table 2 .

Table 2 Mechanical properties of aluminum alloy EN AW1050A H24 from datasheet

\begin{tabular}{|c|c|c|c|c|c|c|}
\hline & $\begin{array}{c}\text { Modulus of } \\
\text { elasticity, } \\
\text { GPa }\end{array}$ & $\begin{array}{l}\text { Proof } \\
\text { stress, } \\
\mathrm{MPa}\end{array}$ & $\begin{array}{l}\text { Tensile } \\
\text { strength, } \\
\mathrm{MPa}\end{array}$ & $\begin{array}{c}\text { Elongation } \\
\text { A, } \%\end{array}$ & $\begin{array}{c}\text { Strength } \\
\text { coefficient } \mathrm{K}, \\
\mathrm{MPa}\end{array}$ & $\begin{array}{c}\text { Strain } \\
\text { hardening } \\
\text { coefficient } \mathrm{n}\end{array}$ \\
\hline Data Sheet Values & 71 & $>85$ & $105-145$ & $3-8$ & & \\
\hline Range for curve fitting & & & & $2 \div 40$ & $140 \div 200$ & $0.05 \div 0.2$ \\
\hline $\begin{array}{l}\text { Calibrated values LS- } \\
\text { Opt \& LS-Dyna }\end{array}$ & & & & 37.9 & 143 & 0.097 \\
\hline
\end{tabular}

The stress-strain relationship was described by the Power-Law material model (Fig. 7). Using the range of material properties from the material data sheet (yield stresses, ultimate strength range and failure strain) the Power-Law parameters such as the strength coefficient $K$, the hardening exponent $\mathrm{n}$ and the failure strain were calibrated using LSOpt system. The input range of the stress-strain curves for the Power-Law models used in LS-Opt is presented in Fig. 7, where the solid line represents the calibrated Power-Law material curve. The Power-Law equation is provided below:

$$
\sigma=K \varepsilon^{n}=143 \varepsilon^{0.097}
$$




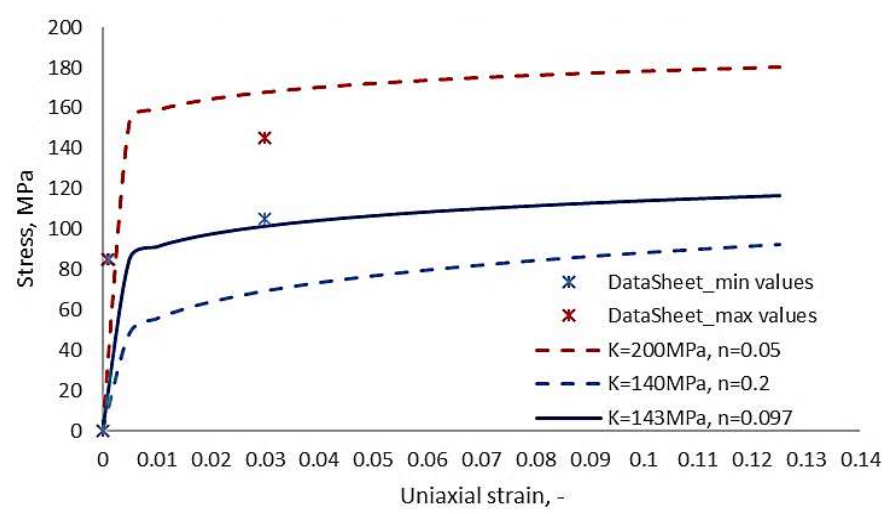

Fig. 7 Stress-strain curves of the Power Law model (dashed curves represent min and max range for the calibration of material model, and solid line - calibrated Power Law function)

Although the fracture strain is stress state dependent [22, 23], the value obtained in the uniaxial test may differ from the failure strain obtained in the cupping test where the biaxial tension is dominant. In case of the cupping test the failure strain is larger than in the uniaxial tensile test. During the incremental forming process the strain path is nonlinear [23], therefore not only the uniaxial failure strain but also the conventional forming limit diagram criterion can cause inaccurate failure prediction.

The explicit FE code LS-Dyna was used to simulate the cupping test and calibrate the material model. The FE model consisted of four parts: a deformable aluminium sheet and a rigid spherical punch, a bottom and a top holder. Initially, the holders were compressed with a force of $10 \mathrm{kN}$. The aluminium sheet was modelled by fully integrated shell elements with 5 integration points through-thickness and a shear correction factor of 0.833 . The size of the shell elements was 0.5 - $1 \mathrm{~mm}$. The FE model (Fig. 8, a) consists of approximately 8500 or 5000 shell elements respectively. The contact between the punch and the aluminum sheet was described using the keyword: *CONTACT_FORMING _ONE_WAY _SURFACE_TO_SURFACE. The speed of the punch was $1 \mathrm{~m} / \mathrm{s}$. The elasto-plastic properties of aluminium were described by the Power Law_Plasticity material model and three parameters were chosen for calibration: strength coefficient $K$, Strain hardening coefficient $n$ and failure strain $\varepsilon_{u}$. The simulation results are presented in Fig. 8, b.

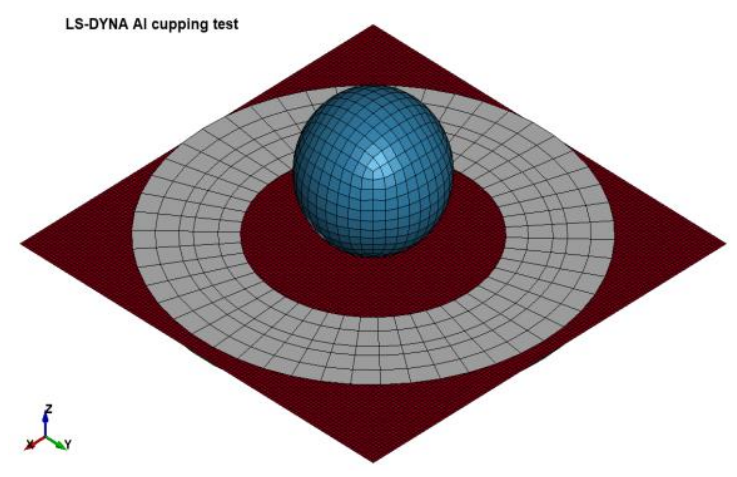

(a)

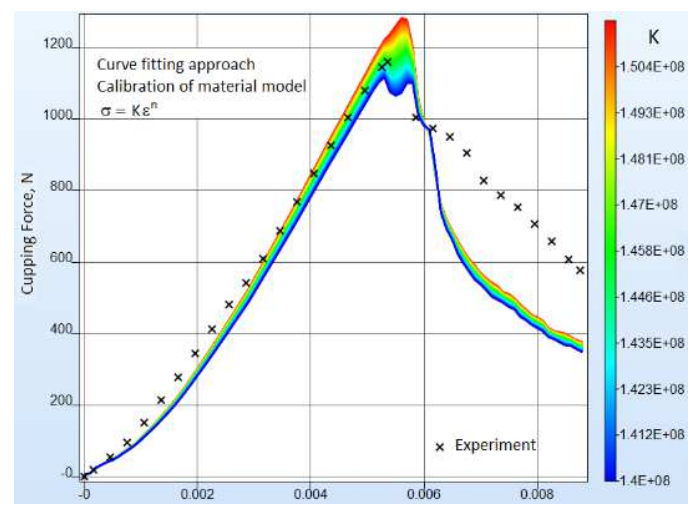

(b)

Fig. 8 Modelling of the cupping test: a numerical model of the cupping test, $\mathbf{b}$ force-displacement plot of the cupping experiment and sensitivity of the simulation results to the strength coefficient $K$ of calibrated Power Law material model (Strain hardening coefficient $n=0.097$ and failure strain $\varepsilon_{u}=0.38$ )

After curve fitting, the experimental force-displacement relation and the FE simulation prediction (Fig. 8, b) correlated well. The spherical punch penetration into an aluminium alloy sheet is illustrated in Fig. 9. Comparing the fracture paths also reveals a common behaviour (Fig. 6, b and Fig. 9). The fracture lines are diagonal and almost symmetrically located between the centre and the corners. Three diagonal fracture lines dominate in the experiments while four diagonal fracture lines are obtained in the simulations. One of the reasons for this could be the anisotropy of the material, which is not evaluated in the FE model. 


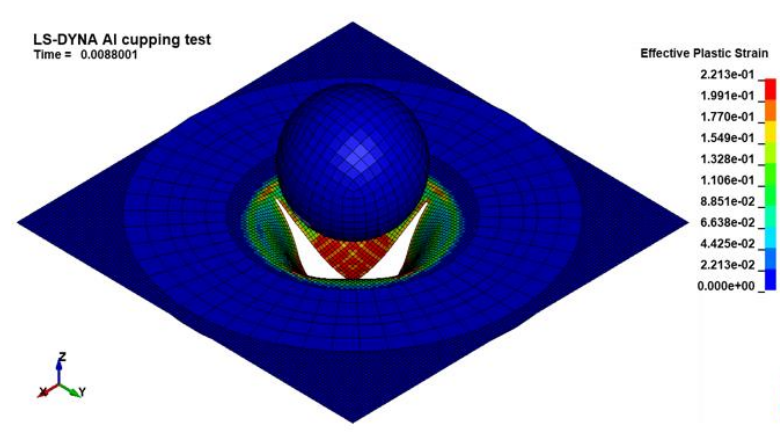

(a)

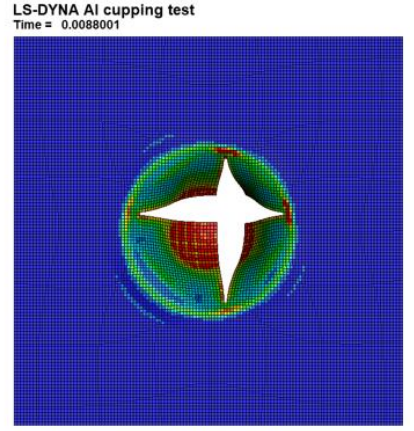

(b)

Fig. 9 Simulated images of a spherical punch piercing an aluminium alloy sheet during the Erichsen test: (a) isometric view and (b) at the bottom of the sheet

\section{Numerical simulations of SPIF process}

As the vibrational excitation of the sheet metal sample was performed by piezo actuators attached to the steel frame, the calculations were also performed by exciting the frame in which the aluminium alloy sheet was mounted. The results of experimental and theoretical studies for the first embedded sheet in steel frame mode, presented in Fig. 10, show a good correlation between the dynamic properties of the Comsol multiphysics $(81.707 \mathrm{~Hz})$ and experimental $(78.8 \mathrm{~Hz})$ models.

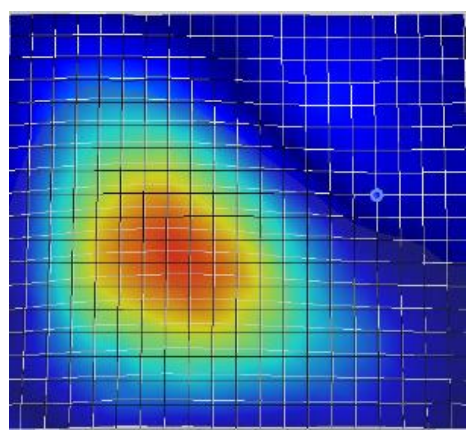

(a)

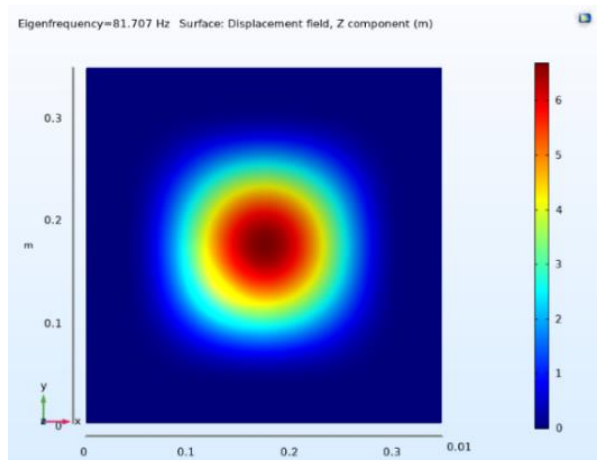

(b)

Fig. 10 The first modes of transverse oscillations of an aluminium alloy sheet fixed in a steel angle: (a) measured at a frequency of $78.8 \mathrm{~Hz}$ and (b) calculated at a frequency of $81,707 \mathrm{~Hz}$ (b)

The SPIF process was modelled using the explicit finite element code LS-Dyna. The SPIF model consisted of two parts: an aluminium sheet and a forming tool (Fig. 11). The aluminium sheet was modelled in the same way as for the cupping test, using fully integrated shell elements with a thickness stretch allowing linear variation of strain through the thickness, 5 integration points through the thickness and a shear correction factor of 0.833 . The size of the shell elements varied between 1 at the centre/contact zone and $5 \mathrm{~mm}$ at the external side of the specimen. The FE model consisted of about 20000 shell elements. The main features of the FE model were copied from the calibrated cupping test model. The elasto-plastic properties of aluminium were described by Power_Law_Plasticity material model. The forming tool was modelled as a rigid body using shell elements. The contact between the bodies was also copied from the cupping test model. The aluminium sheet holder was simulated with fixed nodes ( $25 \mathrm{~mm}$ wide around all 4 edges). Major diameter of the helix was $140 \mathrm{~mm}$. 


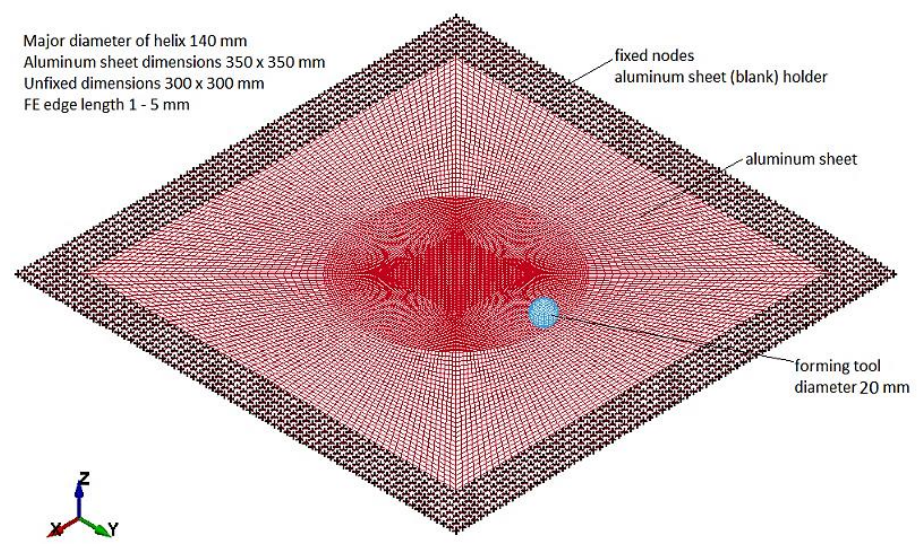

Fig. 11 SPIF simulation model

The load was applied by three functions of tool displacements: $f(x), f(y)$ and $f(z)$. All functions were continuous and varied so that the horizontal displacement per revolution was $0.5 \mathrm{~mm}$ and the vertical displacement per revolution was $0.5 \mathrm{~mm}$. The speed of the forming tool was about $2 \mathrm{~m} / \mathrm{s}$. The trajectories of the forming tool-path are shown in Fig. 12 .

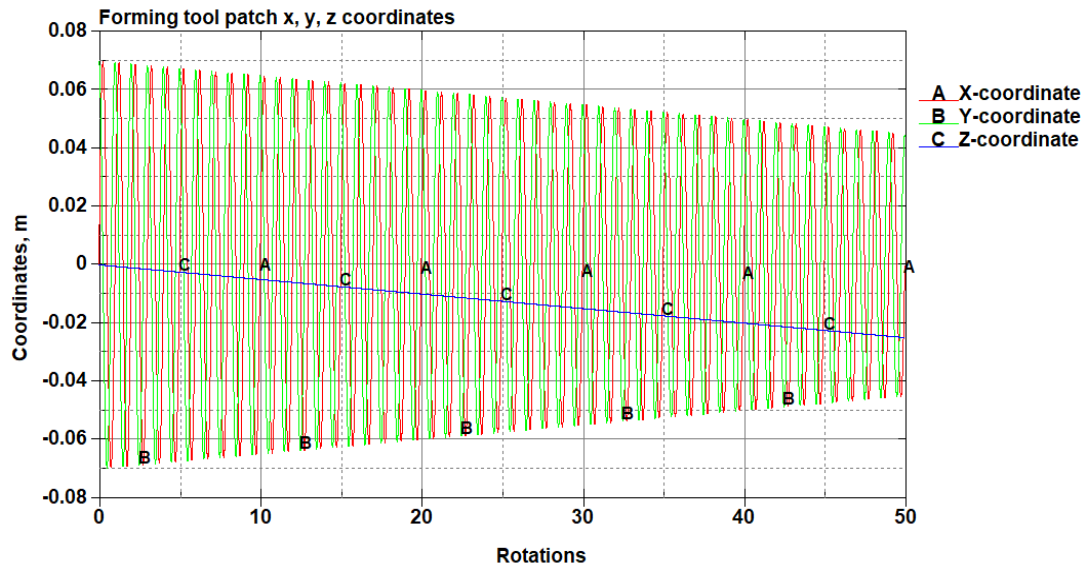

Fig. 12 Loading curves displacements in XYZ directions

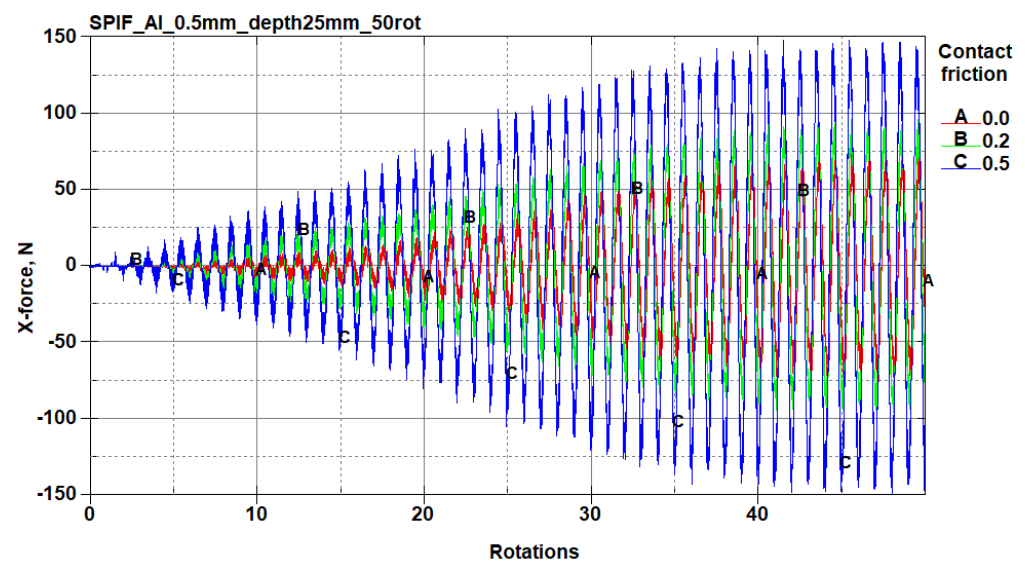

Fig. $13 \mathrm{X}$-contact force vs rotations at the different contact friction coefficients

Various simulations have been carried out with coefficients of friction ranging from 0 to 0.5 . The effect of friction on the horizontal force component is shown in Fig. 13. It can be seen that increasing the coefficient of friction from 0 to 0.5 increases the amplitudes of the $\mathrm{X}$ force component by a factor of approximately 2 . In contrast, the effect on the vertical $\mathrm{Z}$ force component is negligible (Fig. 14, a). The effect of friction on the resultant force is shown in Fig. 14, b. 


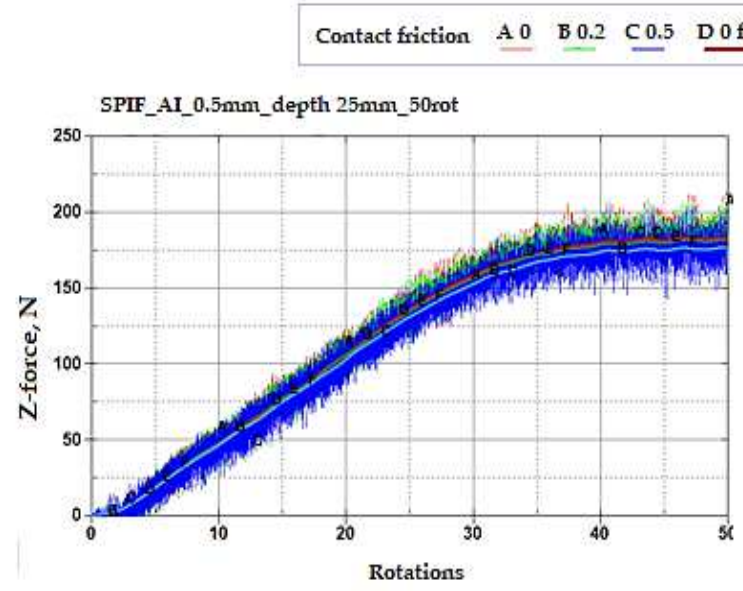

(a)

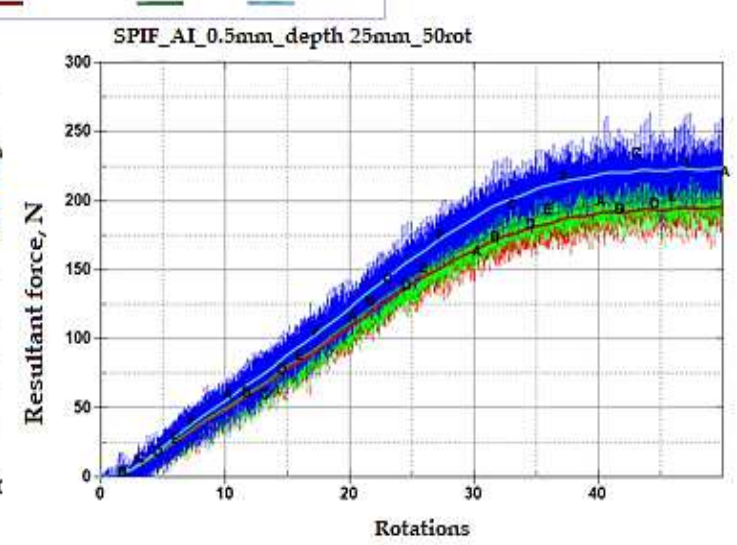

(b)

Fig. 14 Contact force vs rotations for different coefficients of contact friction: (a) z-force (vertical force), (b) resultant force

SPIF is characterized by a reduction in the wall thickness of the final finished part compared to the initial thickness of the sheet metal. The excessive thickness reduction ratio in the deformation zones when the sheet metal is formed separately has a significant effect on the forming limit. The prediction of the thickness of the deformation zone is an important approach to control the thinning ratio. With regard to the object of study, aluminium alloy, the principle of thickness deformation in the SPIF process is presented in Fig. 15.The relationship between the wall thickness and the drawing angle $\alpha$, can be expressed by the sine law used in ISMF:

$$
t_{f}=t_{0} \sin (90-\alpha)
$$

where $t_{0}-$ is the initial thickness, $t_{f}$-is the final thickness, $\alpha$-is the drawing angle between the initial flat surface and the deformed surface.

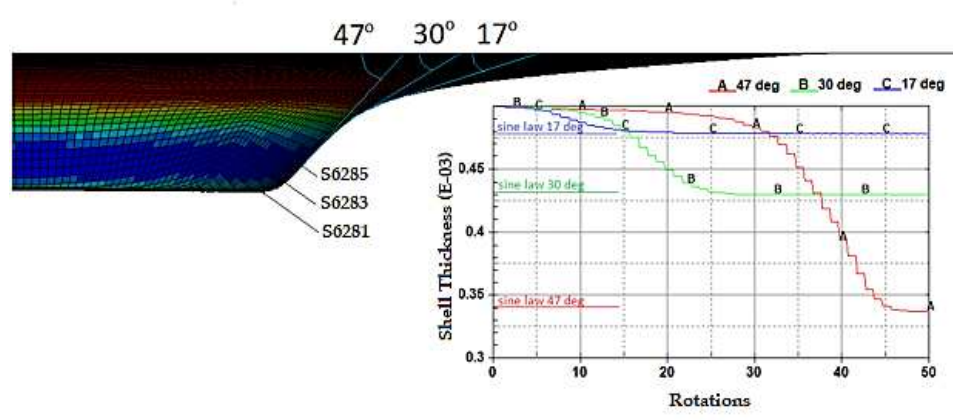

(a)

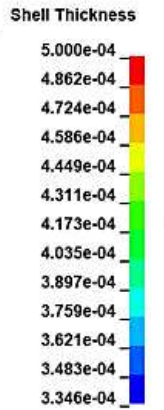

$3.346 \mathrm{e}-04$

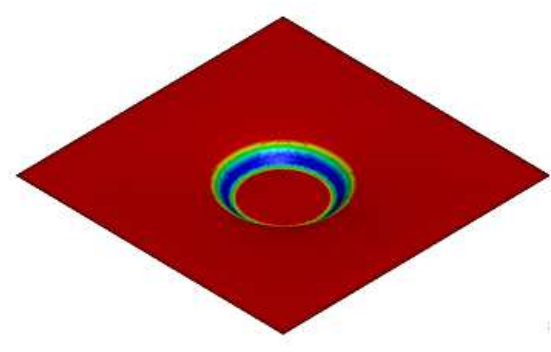

(b)

Fig. 15. Shell thickness reduction: (a) variation at elements which after the forming depth of $25 \mathrm{~mm}$ has the drawing angles of $47^{\circ}, 30^{\circ}$ and $17^{\circ}$, (b) shell thickness distribution after the forming depth of $25 \mathrm{~mm}$

The drawing angle obtained in simulation varies from $47^{\circ}$, therefore, according to the sine law this would lead the reduced thickness up to $0.34 \mathrm{~mm}$. In contrast, the minimum thickness in the simulation was $0.335 \mathrm{~mm}$. The results obtained from the SPIF simulation have good correlation with the sine law. The strain distribution after the forming depth of 25 $\mathrm{mm}$ is presented in Fig. 16

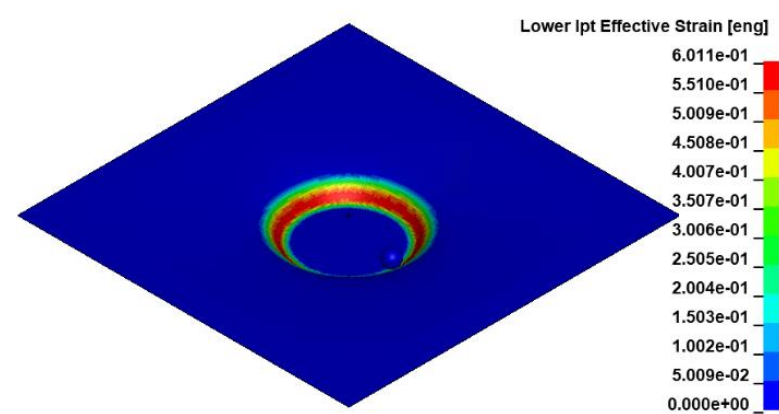

Fig. 16 Effective strain distribution after the forming depth of $25 \mathrm{~mm}$ 
Figures 15 and 16 show that the decrease in sheet thickness during SPIF correlates with effective plastic strain changes. The thinning of the forming sheet is one of the main failure modes in SPIF and is related to the drawing angle $\alpha$ which is one of the geometrical limit of the SPIF process [11]. Fig. 16 shows that the effective strains achieved during the incremental forming (maximum effective strain 60\%) are much higher than the values given in the material data sheet, since the tensile failure strain constitute (3-8)\%, and the values of failure strain calibrated by the cupping test (38\%). This confirms that failure prediction in the incremental forming process is not so straightforward and that other failure criteria should be applied. The SPIF cross sections of the sheet metal per rotation are presented in Fig. 17.

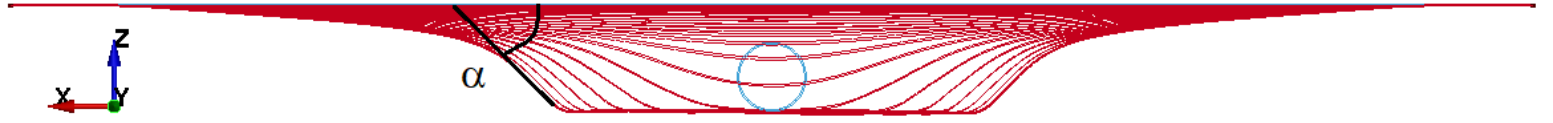

Fig. 17 Cross sections of aluminum forming sheet per rotation

Deformation phenomena during incremental formation are still under discussion. In the absence of direct experimental evidence, some researchers have claimed, that deformation is caused by stretching, while the others state that it is caused by through-thickness shear [24, 25]. If out-of-plane shear dominates, the principal strains are not in the plane [26], therefore the use of shell elements can be limited, as shell elements typically do not allow capturing through-thickness shear properties in simulation. Fully integrated shell elements with a thickness stretch in LS-Dyna allow the capture of through-thickness shear stresses and shear strains. Three elements (S2681, S2683, S2685 see Fig. 15, a) along the x axis were selected to analyse the stresses and strains during the simulation of the SPIF process. The strain distribution through the thickness is shown in Fig. 18.

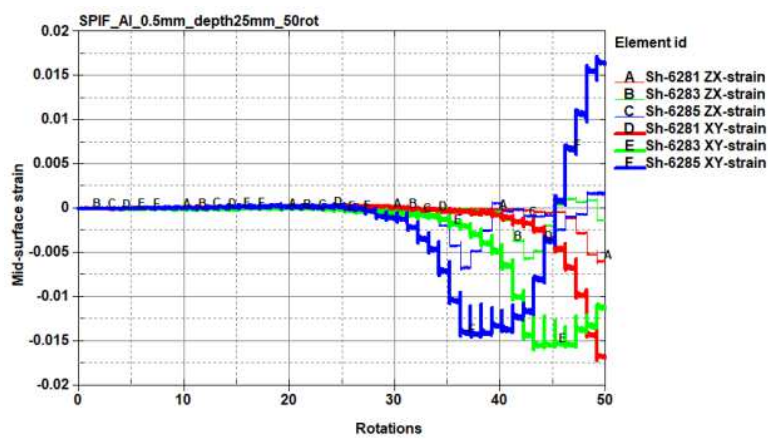

Fig. 18 Variation of in plane and out-off plane shear strains during the SPIF process

The results show that the through-thickness shear strains have significant values comparing to the in-plane (xy) shear strains. In particular, the transverse through-thickness deformation of element No. 6285 accounts for about $50 \%$ of the in-plane shear deformation.

\section{Experimental investigation of SPIF aluminium alloy sheet}

In order to find a more efficient control of the forming force, an attempt was made to excite the aluminium alloy sample by ultrasonic vibrations. The experimental set-up created for this purpose is shown in Fig. 19. An investigated sheet 2 is clamped to the frame 1 and it is excited with two piezoelectric transducers 8 . A power amplifier 7 is used to generate the vibrations in the piezoelectric transducer. The robotic arm 4 incrementally forms the sheet by moving the tool with the sphere attached to it. The tool 3 is specially mounted at $30^{\circ}$ angle to allow the Mechanical momentum gauge (STJ100 (Series BGI Digital Force Gauge with torque sensor STJ100, Mark-10 Corp.) 5 to measure both - the pressing and friction momentum. The gauge is connected to a Keyence LK G3001P sensor controller, which is connected to an oscilloscope (PicoScope 3424) that records the results received from the mechanical moment sensor on a display 6.

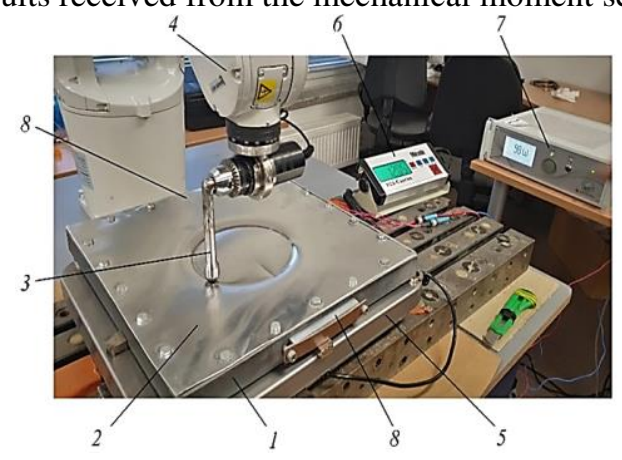

Fig. 19. Experimental set-up of incremental aluminum alloy sheet forming: 1 - frame for aluminum sheet, 2 - aluminum alloy sheet, 3 - forming tool, 4 - robot ABB IRB1200, 5 - scales FCS-4035-150, 6 - display screen of scales, 7 - signal generator, 8 - piezoelectric actuators for ultrasonic excitation 


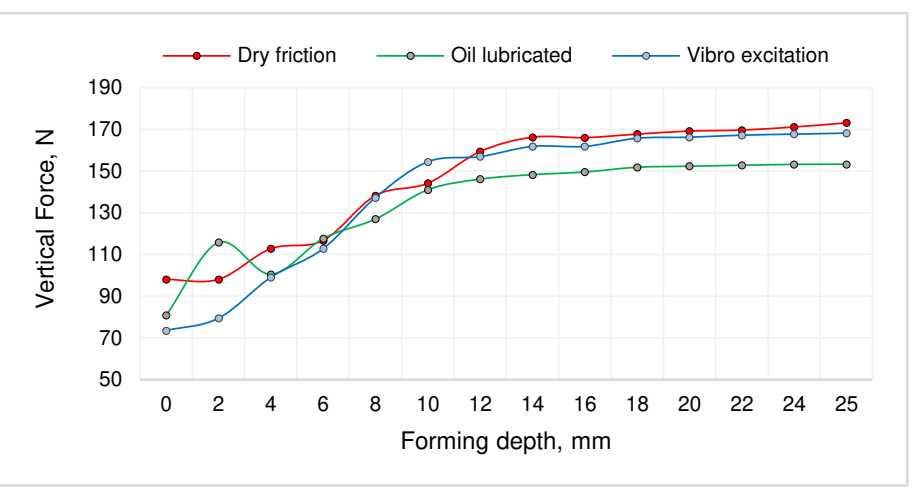

Fig. 20. Graphical representation of vertical force dependency from different friction when sheet thickness is $0.5 \mathrm{~mm}$

The SPIF experiment was performed under dry, lubricated and vibration-excited friction at the tool-sheet metal contact surfaces. Vertical force dependences on the process conditions are depicted in Fig.20.

Accordingly, to verify modelling results, presented in Fig. 8, the puncture forces of the sheet metal during experimental investigation are shown and Fig. 21. It has been observed that at a depth of 0 , the vertical force is equal to $224.04 \mathrm{~N}$; at a depth of 10, the vertical force $778.04 \mathrm{~N}$; at a depth of 16 , the vertical force is $1064 \mathrm{~N}$, but when the depth reaches the value of 15 , the vertical force starts to decrease significantly.

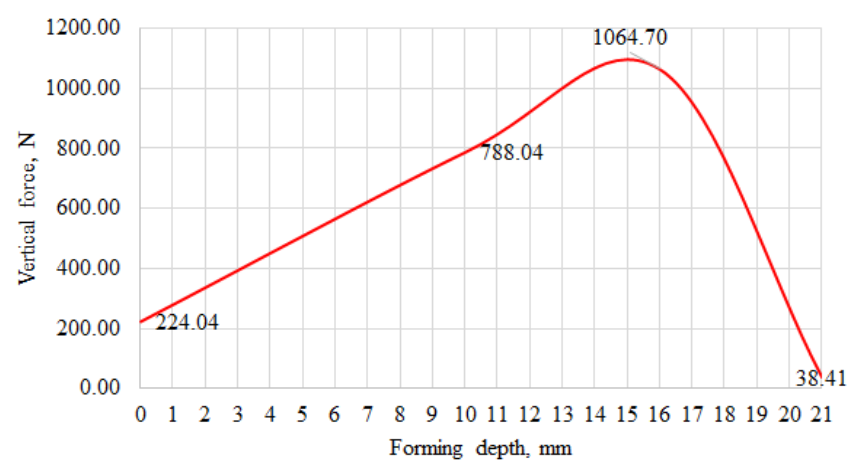

Fig. 21 Graphical representation of the puncture force of an aluminium sheet

Obtained results - curve and magnitude of puncture force showed good correlation with modelling results, presented in Fig. 8, thus theoretical model is verified.

\section{Experimental data exploration}

The data should be analysed to determine whether the data collected are free of data quality problems that could adversely affect the intended prediction models. Common issues such as missing values and outliers should be calculated, because it is impossible to train error-based models with data that contains missing values, furthermore, data that contains outliers can provide incorrect predictions. As depicted in Table 3, there are two types of features: continuous numeric and categorical binary. Two features, namely "Tool end diameter" and "Wall angle" have constant values, therefore they must be eliminated.

Table 3. Parameters for data exploration

\begin{tabular}{clcc}
\hline No. & \multicolumn{1}{c}{ Input parameters } & Units & Value $(\mathbf{m i n}, \mathbf{m a x})$ \\
\hline 1 & Forming depth & $\mathrm{mm}$ & $0 \ldots 26$ \\
2 & Tool End diameter & $\mathrm{mm}$ & $20 \ldots 20$ \\
3 & Step depth, $(\Delta \mathrm{z})$ & $\mathrm{mm}$ & $0.25 \ldots 0.5$ \\
4 & Step width,$(\Delta \mathrm{x})$ & $\mathrm{mm}$ & $0.25 \ldots 0.5$ \\
5 & Wall angle & $\circ$ & $45 \ldots 45$ \\
6 & Sheet thickness, $\left(\Theta,^{\circ}\right)$ & $\mathrm{mm}$ & $0.5 \ldots 0.8$ \\
7 & Tool type & $\mathrm{N}$ & rotating sphere on the end/ not rotating \\
8 & VFC Dry friction & $\mathrm{N}$ & $4.91 \ldots 343.35$ \\
9 & VFC Oil lubricated & & $1.96 \ldots 341.39$ \\
\multicolumn{2}{c}{ Output parameter } & $\mathrm{N}$ & \\
1 & VFC Vibro excitation & & $4.91 \ldots 338.45$ \\
\hline
\end{tabular}


Next, it is reasonable to calculate the correlation coefficients $r$, which indicate the strength of the linear relationship between the two features. The values of Pearson correlation coefficients [28] vary between -1 and 1 , whereas if $r=0$, then the variables have no relationship; the closer the coefficient is to +1 or -1 , the stronger the relationship. The sign indicates whether the relationship is positive or negative, e.g. if $r=1$, then the two features have an ideal positive relationship. A coefficient close to 0 shows a weak correlation. It has been noted that Step depth and Step width have the same values and correlation coefficient $r=1$. It is thus reasonable to remove one of these two features. No outliers or missing data were identified in our dataset.

$$
r=\frac{\sum\left(x_{i}-\bar{x}\right)\left(y_{i}-\bar{y}\right)}{\sqrt{\sum\left(x_{i}-\bar{x}\right)^{2} \sum\left(y_{i}-\bar{y}\right)^{2}}}
$$

were $x_{i}-x$ attribute sample, $y_{i}-y$ attribute sample, $\bar{x}-$ mean of values in $x$ attribute, $\bar{y}-$ mean of values in $y$ attribute,

The correlation coefficient between "Tool type" and the output "VFC Vibro Excitation" is not very significant $r=$ -0.2694 and is actually the lowest value compared to the other features, therefore could be eliminated. After the first iteration of data quality issues management and analysis, our dataset contains 6 raw features obtained directly from data sources. These correlation coefficients $r$ are provided in the Pearson's correlation matrix (heatmap) and presented in Fig. 22 .

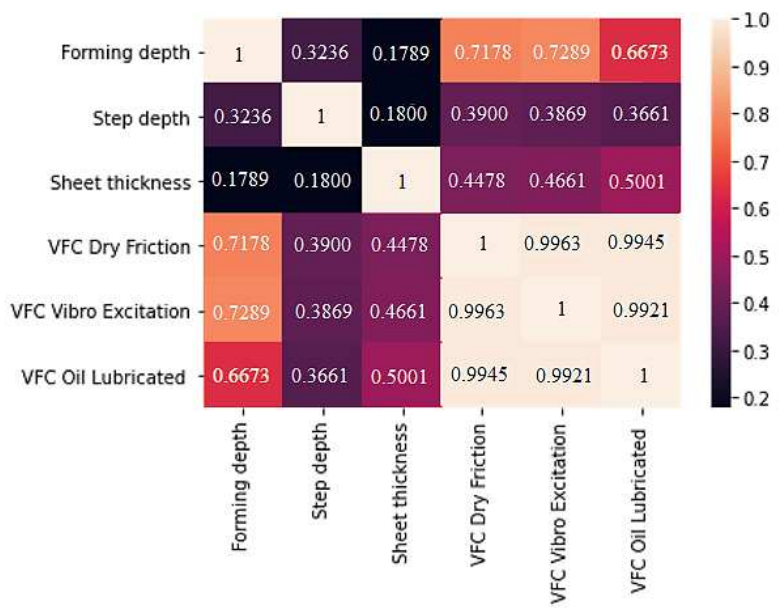

Fig. 22 Pearson correlation matrix and $r$ coefficient

It has been observed that "VFC Dry Friction" has the strongest correlation with the output value, $r=0.9963$. Meanwhile, step depth and sheet thickness, with values $r=0.3869$ and $r=0.4661$ respectively, have shown a moderate correlation with "VFC Vibro Excitation". However, it is reasonable to include all six parameters for the further prediction investigation employing artificial neural networks.

\section{Artificial neural network prediction}

Artificial neural network (ANN) is a system that learns to predict by taking the input data, making a prediction, comparing the prediction to the desired output and adjusting its internal state to predict correctly the next time. Vectors, layers, and linear regression are some of the building blocks of ANN. Each layer transforms the data from the previous layer and can be considered as a feature engineering step, because each layer extracts a particular view of the data presented earlier. Using ANN, the process is starting with some random weights and bias vectors, making a prediction by comparing it to the desired output, and adjusting the vectors to predict more accurately next time. Usually, the process continues until the difference between forecasting and correct targets is minimal. Knowing when to stop training and what minimum error to set is an important aspect of ANN training, largely due to overfitting and underfitting scenarios, which are the biggest causes of bad model performance. The goal of a good ANN model (like any other ML-based model) is to generalize well from the training data to any data from the given problem domain, but in practice it is not very easy to achieve this goal. However, it is very important, since it allows accurate predictions on data that the model has never seen before.

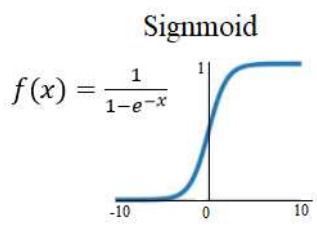

(a)

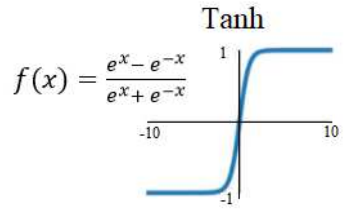

(b)

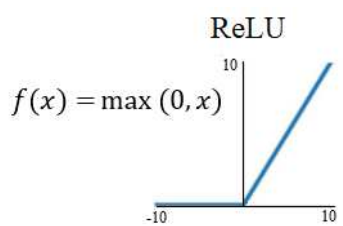

(c) 
Fig. 13. ANN most common activation functions: (a) Sigmoid, (b) Tanh and (c) ReLU

Usually ANN use non-linear activation functions (Fig. 23), which can help the network learn complex data and provide accurate predictions. Sigmoid is a smooth step function, which maps data points into the range [0;1]. The main advantage that this function is simple therefore perfect for binary classification tasks, however it suffers from "vanishing gradients" problem, because its output isn't zero centered. It makes the gradient updates go too far in different directions and makes optimization harder. The updating speed of parameters is very slow and takes very high computational time in hidden layers of ANN. Hyperbolic tangent (Tanh) activation function converts the input value in range $[-1 ; 1]$. Actually higher range of Tanh function is the main difference from the Sigmoid function. Tanh function is more preferred over the Sigmoid function as it allows to achieve better performance for multi-layer ANNs, however it did not solve the vanishing gradient problem that Sigmoid suffered. This problem was solved more effectively with the introduction of rectified linear unit activations: ReLU, Leaky ReLU, ELU. ReLU function became the preferred due to its superiority in handling all drawbacks of Sigmoid and Tanh functions such as "vanishing gradient" problem. Besides, it is computationally efficient, which means that allows network to converge very quickly.

\section{Hyperparameter optimization results}

Hyperparameters, in contrast to machine learning (ML) model parameters, are set manually before the model start training. Hyperparameters cannot be learned within the estimator directly, however model parameters are properties of the training data that are estimated automatically. For example, the minimum leaf size in a decision tree, or kernel scale and function of support vector machine (SVM) are hyperparameters while the weights in an ANN are model parameters learned during training. The choice of hyperparameters in the above models can strongly affect its performance, therefore the optimization process allows to automatically find the optimal combination of hyperparameters for the machine learning algorithm [29]. As the result, an optimal model is provided, which reduces a predefined error value (for example, mean squared error (MSE)) and in turn increases the accuracy on independent data.

In this research, hyperparameter optimization has been performed on ANN model using Bayesian optimization [29]. Two other popular hyperparameter tuning algorithms are grid search and random search. Grid search is the simplest algorithm for hyperparameter tuning, which divides the domain of the hyperparameters into a discrete grid. Theoretically, this algorithm should find the best point in the domain, but practically is not used very often, because it is an exhaustive and time-consuming search. Random search, unlike grid search, does not search solution for every possible combination of hyperparameter values, but tests only a randomly selected subset of these values. Instead of random searching in hyperparameter domain, Bayesian optimization enables intelligence manner of hyperparameters selection, because it uses the results from the previous iteration to decide what is the next set of hyperparameters, which will improve the model performance. Prioritizing of hyperparameters is very efficient and allows to find the best values of hyperparameters' set much faster comparing to both grid search and random search.

For ANN model four hyperparameters have been involved in the optimization process: number of hidden layers, hidden layer size, activation function of hidden layers and regularization strength (Table 4). Three most common activation functions have been analyzed: sigmoid, Tanh and ReLU. The range of regularization strength was chosen based on primary cross-validation results. Value ranges of hidden number layers and hidden layer size were selected according to size of the data set.

Table 4. Hyperparameters of ANN model

\begin{tabular}{llllr}
\hline & \multicolumn{3}{c}{ Hyperparameters of ANN model } \\
\hline & $\begin{array}{l}\text { Number of hidden } \\
\text { layers }\end{array}$ & Hidden layer size & Activation function & Regularization strength \\
Value range & {$[1 ; 3]$} & {$[10 ; 100]$} & Sigmoid, Tanh, ReLU. & {$[0 ; 0.001]$} \\
\hline
\end{tabular}

$K$-cross validation technique [27] has been chosen for ANN model performance assessment. $K$-cross validation splits the data set into $k$ randomly chosen folds of equal size. One subset is used to validate the model trained using the remaining subsets. Such process is repeated $k$ times such that each subset is used exactly once for validation. Finally, the average calculated error (CE) across all $k$ partitions is calculated:

$$
C E=\frac{1}{k} \sum_{i=1}^{k} C E_{i}
$$

Usually Mean Squared Error (MSE) is calculates as CE. In additional to MSE, other accuracy measurement namely, the Root Mean Square Error (RMSE) and the Mean Absolute Error (MAE) were calculated during further experiments.

MSE is a measure representing the average of the squared difference between the real and predicted values in the data set. RMSE is just the square root of the mean square error, the only difference that mean squared error (MSE) measures the variance of the residuals, while RMSE standard deviation of residuals. 


$$
R M S E=\sqrt{M S E}, \quad M S E=\frac{1}{n} \sum_{t=1}^{n}\left|y_{t}-\hat{y}_{t}\right|^{2}
$$

where $n$ - number of time point, $y_{t}-$ is the actual value at a given time period $t$, and $\hat{y}_{t}-$ is the predicted value, $t$ observation in a dataset.

Value of RMSE and MSE penalize large errors. In contrast, MAE is less biased for higher values and usually doesn't penalize large errors. MAE is calculated according to the following formula:

$$
M A E=\frac{1}{n} \sum_{t=1}^{n}\left|y_{t}-\hat{y}_{t}\right|
$$

where $n$ - number of time point, $y_{t}$ - is the actual value at a given time period $t$, and $\hat{y}_{t}-$ is the predicted value.

Fig. 24, a represents the minimum mean squared error (MSE) of GPR algorithm, where red point highlights the iteration that gives the minimum MSE, light blue point represents computed MSE value during optimization process by varying GPR hyperparameters. Dark blue points represent observed minimum error minMSE detected up to current (including current as well) observation:

$$
\operatorname{minMSE} E_{i}=\min \left(\min M S E^{1}, \operatorname{minMSE} E^{i-2}, \min M S E^{i-1}, \ldots, \min M S E^{i}\right), i=\overline{1, n},
$$

where $n$ is number of iterations.

Estimated minimum MSE values vary in the range [108.142; 20.573], observed minMSE vary from 96.883 to 20.573 Fig. 24, b shows cross validation results depicting the predicted value of VFC vibro excitation against the real (true) values. Errors are displayed in vertical red dash lines, but due to the very small error values, majority of true and predicted value points overlap.

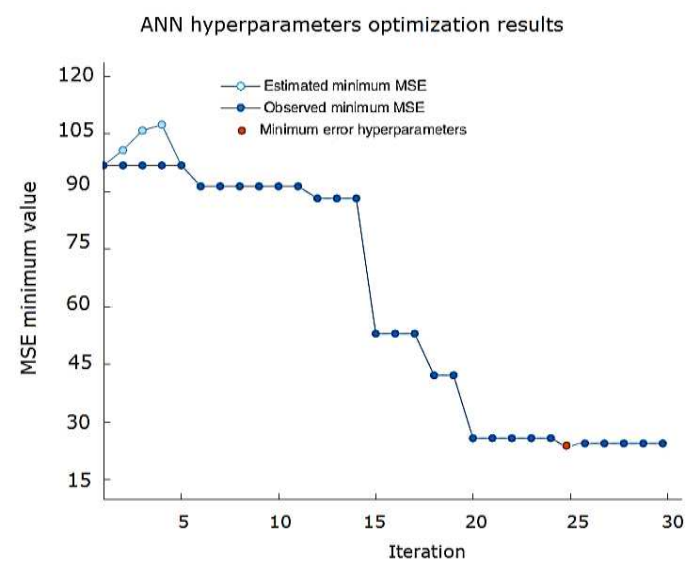

(a)

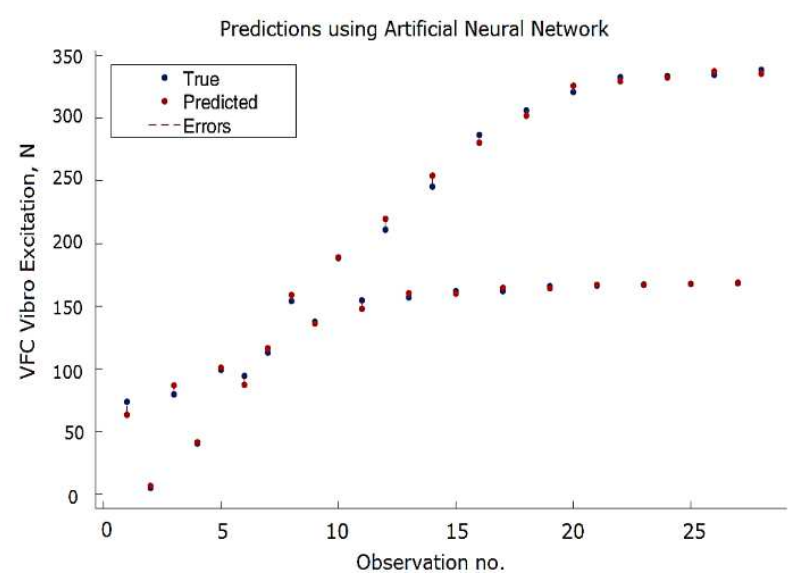

(b)

Fig. 24 Minimum MSE during ANN hypermeters': (a) optimization process and (b) testing data results of ANN model

ANN approach provides very good prediction accuracy and the best results with RMSE $=4.5337, \mathrm{MSE}=20.573$ and MAE $=3.528$ were gained using one hidden layer neural network with ReLU activation function, 12 neurons and 0 regularization strength (Fig. 25) in the 25-th iteration of the hyperparameter optimization process. The optimal ANN architecture includes 3 layers in total: input layer with 5 neurons, hidden layer with 12 neurons and output layer with one neurons providing real type value.

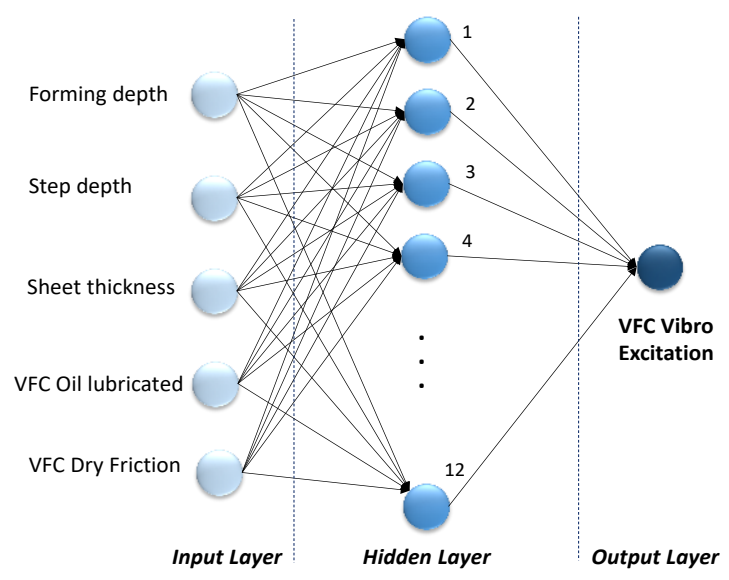


Fig. 25 ANN model architecture providing the best MSE value

In order to estimate how well the regression predictions approximate the real data, an $R^{2}$ (coefficient of determination) regression score was calculated. $R^{2}$ is a statistical measure defining the closeness of the data to the fitted regression line. An $R^{2}$ of 1 indicates that the regression predictions perfectly fit the data.

$$
R^{2}=\frac{S S R}{S S T}=\frac{\sum_{i=1}^{m}\left(y_{i}-\widehat{y_{l}}\right)^{2}}{\sum_{i=1}^{m}\left(y_{i}-\bar{y}\right)^{2}}
$$

where $S S R$ is the sum of squares of residuals, $S S T$ - total sum of squares, $y_{i}-$ actual value, $\widehat{y}_{l}-$ predicted value and $\bar{y}$ mean value.

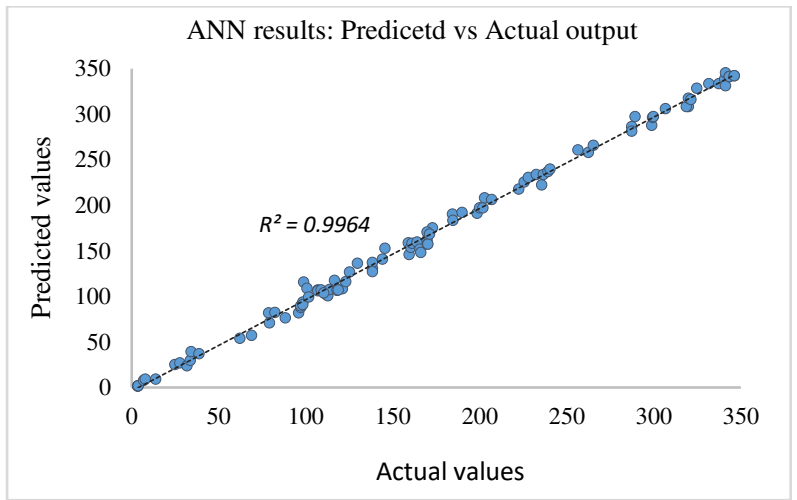

Fig. 26. R-squared assessing Goodness-of-Fit in a ANN Model

The $R$ - squared is always between 0 and $100 \%$ (or 0 and 1.0). The more $R$ - squared, the better the model. The goal is not to maximize the $R$-squared because the stability and adaptability of the model are equally important. When checking the adjusted $R$-squared value, it is best if the values of the $R$-squared and adjusted $R$-squared values are close to each other. If this is not the case, the analyst may overfit the model and may need to remove insignificant variables from the model. ANN have been identified as a very efficient method for developing VFC Vibro excitation prediction models, because $R^{2}$ is equal to 0.9964 (Fig. 26).

All experiments with the ANN model were implemented in Matlab R2020a and run on a PC (Dell Allienware) with NVidia GeForce GTX 980M with 4GB GDDR5 graphics and 4GB of GDDR5 standard memory.

\section{Conclusions}

In the paper, numerical, experimental and artificial intelligence methods are used to analyze the deformation forces of a single point robotized incremental forming of aluminium alloy sheet. An innovative method for reducing the frictional force between the forming tool and the sheet surface by excitation of the workpiece with high frequency oscillations in two orthogonal directions in the sheet plane is proposed. The coefficient of friction between the dry sheet surface and the tool has been found to be equal to the coefficient of friction between the lubricated surfaces, which speeds up the product's way to market and makes the process more environmentally friendly. Thanks to the numerical FEM, the decreasing tendencies of the sheet cross section depending on the degree of plastic deformation were investigated.

The ANN model was developed and applied to predict the force of the forming process when the workpiece is excited. Bayesian hyperparameter optimization was performed in the study to obtain the best ANN architecture and to achieve the best possible result with this architecture by tuning four network hyperparameters. The experimental results showed that the use of a single hidden layer (with 12 neurons) ANN in sufficient to achieve $\mathrm{RMSE}=4.5337$ and $R^{2}=0.9964$.

Funding This research was funded by the European Regional Development Fund according to the supported activity No. 01.2.2- LMT-K-718 under the project No. DOTSUT-234.

Consent to participate It is confirmed that all the authors are aware and satisfied with the authorship order and correspondence of the paper.

Consent for publication All the authors are satisfied that the last revised version of the paper is published without any change.

Conflict of interest The authors declare no competing interests.

Availability of data and material The data required to reproduce these findings cannot be shared at this time as the data also forms part of an ongoing study.

Code Availability There are no software codes to share.

Ethics approval Not applicable.

Authors' contributions Vytautas Ostasevicius: supervision, ideas generation, investigation, funding acquisition, writing. 
Agne Paulauskaite-Taraseviciene: experimental data exploration, artificial neural network prediction, Hyperparameter optimization results, review and editing.

Ieva Paleviciute: investigation, data acquisition, formal analysis.

Vytautas Jurenas: experimental research, methodology, resources, investigation.

Paulius Griskevicius: modelling, theoretical research, visualization.

Darius Eidukynas: experimental research, data acquisition, visualization, review and editing.

Laura Kizauskiene: artificial neural network prediction, writing, review and editing.

\section{References}

1. Callegari, M., Amodio, D., Ceretti, E., \& Giardini, C. (2006). Sheet incremental forming: advantages of robotised cells vs. CNC machines. In Industrial Robotics: Programming, Simulation and Applications. IntechOpen.493-514. https://doi.org/10.5772/4913

2. Belchior, J., Leotoing, L., Guines, D., Courteille, E., \& Maurine, P. (2014). A process/machine coupling approach: application to robotized incremental sheet forming. Journal of Materials Processing Technology, 214(8), $1605-1616$. https://doi.org/10.1016/j.jmatprotec.2014.03.005

3. Belchior, J., Guillo, M., Courteille, E., Maurine, P., Leotoing, L., \& Guines, D. (2013). Off-line compensation of the tool path deviations on robotic machining: Application to incremental sheet forming. Robotics and ComputerIntegrated Manufacturing, 29(4), 58-69. https://doi.org/10.1016/j.rcim.2012.10.008

4. Li, P., He, J., Liu, Q., Yang, M., Wang, Q., Yuan, Q., \& Li, Y. (2017). Evaluation of forming forces in ultrasonic incremental sheet metal forming. Aerospace Science and Technology, 63, 132-139. https://doi.org/10.1016/j.ast.2016.12.028

5. Li, P. Y., Liu, Q., An, W. R., \& Li, S. J. (2016). Finite Element Simulation of Ultrasonic Incremental Forming. In Materials Science Forum (Vol. 836, pp. 452-461). Trans Tech Publications Ltd. https://doi.org/10.4028/www.scientific.net/MSF.836-837.452

6. Hadoush, A., \& van den Boogaard, A. H. (2009). Substructuring in the implicit simulation of single point incremental sheet forming. International Journal of Material Forming, 2(3), 181-189. https://doi.org/10.1007/s12289-009-0402-3

7. Mulay, A., Ben, S., Syed, I., \& Ben, A. (2017). Artificial neural network modeling of quality prediction of a single point incremental sheet forming process. Advanced Science and Technology Letters, 147, 224-250. http://dx.doi.org/10.14257/astl.2017.147.34

8. Verleysen, P., Peirs, J., Van Slycken, J., Faes, K., \& Duchene, L. (2011). Effect of strain rate on the forming behaviour of sheet metals. Journal of Materials Processing Technology, 211(8), 1457-1464. https://doi.org/10.1016/j.jmatprotec.2011.03.018

9. Wu, R., Hu, Q., Li, M., Cai, S., \& Chen, J. (2021). Evaluation of the forming limit of incremental sheet forming based on ductile damage. Journal of Materials Processing Technology, 287, 116497. https://doi.org/10.1016/j.jmatprotec.2019.116497

10. Guzmán, C. F., Yuan, S., Duchêne, L., Flores, E. I. S., \& Habraken, A. M. (2018). Damage prediction in single point incremental forming using an extended Gurson model. International Journal of Solids and Structures, 151, 45-56. https://doi.org/10.1016/j.ijsolstr.2017.04.013

11. Bouffioux, C., Lequesne, C., Vanhove, H., Duflou, J. R., Pouteau, P., Duchêne, L., \& Habraken, A. M. (2011). Experimental and numerical study of an AlMgSc sheet formed by an incremental process. Journal of Materials Processing Technology, 211(11), 1684-1693. https://doi.org/10.1016/j.jmatprotec.2011.05.010

12. Ambrogio, G., Filice, L., Guerriero, F., Guido, R., \& Umbrello, D. (2011). Prediction of incremental sheet forming process performance by using a neural network approach. The International Journal of Advanced Manufacturing Technology, 54(9), 921-930. https://doi.org/10.1007/s00170-010-3011-X

13. Centeno, G., Bagudanch, I., Martínez-Donaire, A. J., Garcia-Romeu, M. L., \& Vallellano, C. (2014). Critical analysis of necking and fracture limit strains and forming forces in single-point incremental forming. Materials \& Design, 63, 20-29. https://doi.org/10.1016/j.matdes.2014.05.066

14. Varthini, R., Gandhinathan, R., Pandivelan, \& Jeevanantham, A, K. (2014). Modelling And Optimization Of Process Parameters Of The Single Point Incremental Forming Of Aluminium 5052 Alloy Sheet Using Genetic AlgorithmBack Propagation Neural Network. International Journal of Mechanical and Production Engineering (IJMPE), 2(5), 55-62.

15. Oraon, M., \& Sharma, V. (2018). Predicting force in single point incremental forming by using artificial neural network. International Journal of Engineering, 31(1), 88-95. doi: 10.5829/ije.2018.31.01a.13

16. Hartmann, C., Opritescu, D., \& Volk, W. (2019). An artificial neural network approach for tool path generation in incremental sheet metal free-forming. Journal of Intelligent Manufacturing, 30(2), 757-770. https://doi.org/10.1007/s10845-016-1279-X

17. Mulay, A., Ben, S., Syed, I., \& Ben, A. (2017). Artificial neural network modeling of quality prediction of a single point incremental sheet forming process. Advanced Science and Technology Letters, 147, $224-250$. http://dx.doi.org/10.14257/astl.2017.147.34

18. Ostasevicius V, Jurenas V, Grigaliunas V, Eidukynas D, Bubulis A, Paleviciute (2020) Incremental forming machine for sheet metal parts. Patent in LT patent bureau, application no.: LT2020 516. 
19. Bhattacharya, A., Maneesh, K., Venkata Reddy, N., \& Cao, J. (2011). Formability and surface finish studies in single point incremental forming. Journal of manufacturing science and engineering, 133(6). https://doi.org/10.1115/1.4005458

20. Stoughton, T. B., \& Yoon, J. W. (2011). A new approach for failure criterion for sheet metals. International Journal of Plasticity, 27(3), 440-459. https://doi.org/10.1016/j.ijplas.2010.07.004

21. Jawale, K., Duarte, J. F., Reis, A., \& Silva, M. B. (2018). Microstructural investigation and lubrication study for single point incremental forming of copper. International Journal of Solids and Structures, 151, 145-151. https://doi.org/10.1016/j.ijsolstr.2017.09.018

22. Gorji, M., Berisha, B., Manopulo, N., \& Hora, P. (2016). Effect of through thickness strain distribution on shear fracture hazard and its mitigation by using multilayer aluminum sheets. Journal of Materials Processing Technology, 232, 19-33. https://doi.org/10.1016/j.jmatprotec.2016.01.014

23. Dietsch, P., Tihay, K., Bui-Van, A., \& Cornette, D. (2017). Methodology to assess fracture during crash simulation: fracture strain criteria and their calibration. Metallurgical Research \& Technology, 114(6), 607. https://doi.org/10.1051/metal/2016065

24. Silva, M. B., Skjødt, M., Atkins, A. G., Bay, N., \& Martins, P. A. F. (2008). Single-point incremental forming and formability-failure diagrams. The Journal of Strain Analysis for Engineering Design, 43(1), 15-35. https://doi.org/10.1243/03093247JSA340

25. Allwood, J. M., Shouler, D. R., \& Tekkaya, A. E. (2007). The increased forming limits of incremental sheet forming processes. In Key Engineering Materials (Vol. 344, pp. 621-628). Trans Tech Publications Ltd. https://doi.org/10.4028/www.scientific.net/KEM.344.621

26. Emmens, W. C., \& van den Boogaard, A. H. (2007). Strain in shear, and material behaviour in incremental forming. In Key engineering materials (Vol. 344, pp. 519-526). Trans Tech Publications Ltd. https://doi.org/10.4028/www.scientific.net/KEM.344.519

27. Browne, M.W (2000). Cross-Validation Methods. In journal of Mathematical Psychology, (Vol. 44 (1), pp.108-132), https://doi.org/10.1006/jmps.1999.1279

28. Benesty J., Chen J., Huang Y., Cohen I. (2009) Pearson Correlation Coefficient (pp. 1-4). In: Noise Reduction in Speech Processing. Springer Topics in Signal Processing, vol 2. Springer. https://doi.org/10.1007/978-3-642-00296$\underline{0 \_}$

29. Y. Li., Shami A (2020). On hyperparameter optimization of machine learning algorithms: Theory and practice, In Neurocomputing, (Vol. 415, pp. 295-316). https://doi.org/10.1016/j.neucom.2020.07.061 